\title{
On the quartic Gauss sums and their recurrence property
}

\section{Shimeng Shen and Wenpeng Zhang*}

\section{"Correspondence:}

wpzhang@nwu.edu.cn

School of Mathematics, Northwest

University, Xi'an, Shaanxi, P.R. China

\begin{abstract}
The main purpose of this paper is, using the method of trigonometric sums and the properties of Gauss sums, to study the computational problem of one kind of congruence equation modulo an odd prime and give some interesting fourth-order linear recurrence formulas.
\end{abstract}

MSC: 11L05

Keywords: quartic Gauss sums; trigonometric sums; recurrence formulas

\section{Introduction}

Let $q \geq 3$ be a positive integer. For any positive integer $k$ and integer $m$, the $k$ th Gauss sums $G(m, k ; q)$ are defined as

$$
G(m, k ; q)=\sum_{a=1}^{q} e\left(\frac{m a^{k}}{q}\right)
$$

where $e(y)=e^{2 \pi i y}$.

Concerning these sums, many authors had studied their properties and obtained a series of interesting results, see [1,2]. In fact, from Weil's important work [1], one can get the upper bound estimate

$$
\left|\sum_{a=1}^{p-1} \chi(a) e\left(\frac{m a^{k}}{p}\right)\right| \ll_{k} \sqrt{p},
$$

where $p$ is an odd prime, $\chi$ denotes a Dirichlet character $\bmod p$ and $\ll_{k}$ denotes the Big O notation dependent on $k$.

We also mention that the fourth power mean value of $G(m, k ; q)$ was well explored by Zhang and Liu [2], in which some sharp asymptotic formulas can be found.

On the other hand, Yang and Tang [3] studied a number of solutions of the congruence equation

$$
x^{2}+y^{2} \equiv c \bmod n \quad \text { with }(x y, n)=1
$$

and gave an exact computational formula for it.

(c) The Author(s) 2017. This article is distributed under the terms of the Creative Commons Attribution 4.0 International License (http://creativecommons.org/licenses/by/4.0/), which permits unrestricted use, distribution, and reproduction in any medium, provided you give appropriate credit to the original author(s) and the source, provide a link to the Creative Commons license, and indicate if changes were made. 
Let $s$ be a positive integer and $p$ be an odd prime with $p \equiv 1 \bmod 3$. Let $M_{s}$ denote a number of solutions of the equation

$$
X_{1}^{3}+X_{2}^{3}+X_{3}^{3}+\cdots+X_{s}^{3}=0
$$

in the finite field $G F(p)$, and let $U_{s}=M_{s}-p^{s-1}$. Chowla et al. [4] proved that $U_{s}$ satisfies the linear recurrence

$$
U_{s}-3 p U_{s-2}-p d U_{s-3}=0
$$

where $U_{1}=0, U_{2}=2 p-2$ and $U_{3}=(p-1) d$ with $d$ being uniquely determined by $4 p=$ $d^{2}+27 b^{2}$ and $d \equiv 1 \bmod 3$.

Some related results can also be found in [5-7].

Now we consider a similar problem: Let $n$ be a positive integer and $p$ be an odd prime with $p \equiv 1 \bmod 4$. Let $M_{n}(p)$ denote a number of solutions of the congruence equation

$$
x_{1}^{4}+x_{2}^{4}+\cdots+x_{n-1}^{4}+x_{n}^{4} \equiv 0 \bmod p,
$$

where $0 \leq x_{i} \leq p-1, i=1,2, \ldots, n$.

It is natural to ask whether there exists an exact computational formula for $M_{n}(p)$ when $n$ is a positive integer and $p$ is an odd prime?

As far as we know, it seems that no one has studied this problem yet, at least we have not seen any related result before. The problem is interesting because it can help us to understand more accurate information of the quartic Gauss sums.

In this paper, we shall use the method of trigonometric sums and the properties of Gauss sums to study this problem and give some interesting computational formulas. For the sake of convenience, first we let $B(p)=\sum_{a=1}^{p-1}\left(\frac{a+\bar{a}}{p}\right), \bar{a}$ denotes the solution of the equation $a x \equiv 1 \bmod p$, and $\left(\frac{*}{p}\right)$ denotes the Legendre symbol $\bmod p$. Then we have the following theorem.

Theorem 1 Let $p=8 k+5$ be a prime, $U_{n}(p)=M_{n}(p)-p^{n-1}$. Then, for any positive integer $n \geq 5$, we have the fourth-order linear recurrence formula

$$
U_{n}(p)=-2 p U_{n-2}(p)+4 p B(p) U_{n-3}(p)-\left(9 p^{2}-p B^{2}(p)\right) U_{n-4}(p),
$$

where the first four terms are $U_{1}(p)=0, U_{2}(p)=-(p-1), U_{3}(p)=3(p-1) B(p)$ and $U_{4}(p)=$ $-7 p(p-1)+(p-1) B^{2}(p)$.

Theorem 2 Let $p=8 k+1$ be a prime, $U_{n}(p)=M_{n}(p)-p^{n-1}$. Then, for any positive integer $n \geq 5$, we have the fourth-order linear recurrence formula

$$
U_{n}(p)=6 p U_{n-2}(p)+4 p B(p) U_{n-3}(p)-\left(p^{2}-p B^{2}(p)\right) U_{n-4}(p)
$$

where the first four terms are $U_{1}(p)=0, U_{2}(p)=3(p-1), U_{3}(p)=3(p-1) B(p)$ and $U_{4}(p)=$ $17 p(p-1)+(p-1) B^{2}(p)$.

From these theorems we may immediately deduce the following two corollaries. 
Corollary 1 Let $p=8 k+5$ be an odd prime. Then we have

$$
M_{4}(p)=p^{3}+(p-1) B^{2}(p)-7 p(p-1), \quad M_{5}(p)=p^{4}-10 p(p-1) B(p) .
$$

Corollary 2 Let $p=8 k+1$ be an odd prime. Then we have

$$
M_{4}(p)=p^{3}+(p-1) B^{2}(p)+17 p(p-1), \quad M_{5}(p)=p^{4}+30 p(p-1) B(p) .
$$

Note the estimate for character sums

$$
|B(p)|=\left|\sum_{a=1}^{p-1}\left(\frac{a+\bar{a}}{p}\right)\right| \leq 2 \sqrt{p},
$$

from Corollary 1 and Corollary 2 we can also deduce the following corollary.

Corollary 3 Let $p$ be an odd prime with $p \equiv 1 \bmod 4$. Then we have the asymptotic formulas

$$
M_{4}(p)=p^{3}+O\left(p^{2}\right) \quad \text { and } \quad M_{5}(p)=p^{4}+O\left(p^{\frac{5}{2}}\right) .
$$

Remark Let $p$ be an odd prime with $p \equiv 1 \bmod 4$, it is clear that we have the identity (see Theorems 4-11 in [8])

$$
p=\left(\frac{1}{2} \sum_{a=1}^{p-1}\left(\frac{a+\bar{a}}{p}\right)\right)^{2}+\left(\frac{1}{2} \sum_{a=1}^{p-1}\left(\frac{a+r \bar{a}}{p}\right)\right)^{2}=\alpha^{2}+\beta^{2},
$$

where $r$ is a quadratic non-residue $\bmod p$, that is to say, $\left(\frac{r}{p}\right)=-1$. The above identity implies that $|B(p)|$ is a constant depending only on $p$ and $|B(p)| \leq 2 \sqrt{p}$.

For prime $p=4 k+3$ and positive $b$ with $1 \leq b \leq p-1$, we have the identity

$$
A(b)=\sum_{a=0}^{p-1} e\left(\frac{b a^{4}}{p}\right)=i\left(\frac{b}{p}\right) \sqrt{p}, \quad i^{2}=-1 .
$$

It is very easy to prove that $M_{2 n-1}(p)=p^{2 n-2}$ and

$$
M_{2 n}(p)=p^{2 n-1}+(-1)^{n} p^{n-1}(p-1)
$$

\section{Several lemmas}

In this section, we give some lemmas which are necessary in the proofs of our theorems. Hereinafter, we shall use some properties of the classical Gauss sums, all of them can be found in reference [9], so they will not be repeated here. First we have the following lemma.

Lemma 1 Let $p$ be an odd prime with $p \equiv 1 \bmod 4,\left(\frac{*}{p}\right)=\chi_{2}$ denotes the Legendre symbol $\bmod p$. Then, for any integer $b$ with $(b, p)=1$, we have the identities

$$
\text { (I) } A(b)=\sum_{a=0}^{p-1} e\left(\frac{b a^{4}}{p}\right)=\left(\frac{b}{p}\right) \sqrt{p}+\sum_{a=1}^{p-1}\left(\frac{a}{p}\right) e\left(\frac{b a^{2}}{p}\right) \text {; }
$$


(II) $A^{2}(b)=R(p) \cdot p+2\left(\frac{b}{p}\right) \sqrt{p}\left(\sum_{a=1}^{p-1}\left(\frac{a}{p}\right) e\left(\frac{b a^{2}}{p}\right)\right)+\left(\frac{b}{p}\right) \sqrt{p} \sum_{a=1}^{p-1}\left(\frac{a+\bar{a}}{p}\right)$,

where $R(p)=-1$, if $p=8 k+5 ;$ and $R(p)=3$, if $p=8 k+1$.

Proof From the definition of the quadratic residue $\bmod p$ and the properties of the Legendre symbol, we have

$$
\begin{aligned}
A(b) & =\sum_{a=0}^{p-1} e\left(\frac{b a^{4}}{p}\right)=1+\sum_{a=1}^{p-1} e\left(\frac{b a^{4}}{p}\right)=1+\sum_{a=1}^{p-1}\left(1+\chi_{2}(a)\right) e\left(\frac{b a^{2}}{p}\right) \\
& =\sum_{a=0}^{p-1} e\left(\frac{b a^{2}}{p}\right)+\sum_{a=1}^{p-1} \chi_{2}(a) e\left(\frac{b a^{2}}{p}\right) .
\end{aligned}
$$

Since $p \equiv 1 \bmod 4$, from [9] (see formula (29) of Section 9.10) we know that

$$
\sum_{a=0}^{p-1} e\left(\frac{b a^{2}}{p}\right)=\left(\frac{b}{p}\right) \sum_{a=0}^{p-1} e\left(\frac{a^{2}}{p}\right)=\left(\frac{b}{p}\right) \sqrt{p}
$$

From (2) and (3) we may immediately deduce formula (I) of Lemma 1.

Now we prove formula (II) of Lemma 1. It is clear that from (I) we have

$$
A^{2}(b)=p+2\left(\frac{b}{p}\right) \sqrt{p}\left(\sum_{a=1}^{p-1}\left(\frac{a}{p}\right) e\left(\frac{b a^{2}}{p}\right)\right)+\left(\sum_{a=1}^{p-1}\left(\frac{a}{p}\right) e\left(\frac{b a^{2}}{p}\right)\right)^{2} .
$$

From the properties of the reduced residue system $\bmod p$ and (3), we have

$$
\begin{aligned}
\left(\sum_{a=1}^{p-1}\left(\frac{a}{p}\right) e\left(\frac{b a^{2}}{p}\right)\right)^{2}= & \sum_{x=1}^{p-1} \sum_{y=1}^{p-1}\left(\frac{x y}{p}\right) e\left(\frac{b\left(x^{2}+y^{2}\right)}{p}\right) \\
= & \sum_{x=1}^{p-1}\left(\frac{x}{p}\right) \sum_{y=1}^{p-1} e\left(\frac{b y^{2}\left(x^{2}+1\right)}{p}\right) \\
= & (p-1) \sum_{x=1}^{p-1}\left(\frac{x}{p}\right) \\
& +\sum_{\substack{x=1 \\
\left(x^{2}+1, p\right)=1}}^{p-1}\left(\frac{x}{p}\right)\left(\sum_{y=0}^{p-1} e\left(\frac{b y^{2}\left(x^{2}+1\right)}{p}\right)-1\right) \\
= & p \sum_{x=1}^{p-1}\left(\frac{x}{p}\right)-\sum_{x=1}^{p-1}\left(\frac{x}{p}\right)+\left(\frac{b}{p}\right) \sqrt{p} \sum_{x=1}^{p-1}\left(\frac{x\left(x^{2}+1\right)}{p}\right) \\
= & I(p) p+\left(\frac{b}{p}\right) \sqrt{p} \sum_{a=1}^{p-1}\left(\frac{a+\bar{a}}{p}\right),
\end{aligned}
$$

where $I(p)=-2$, if $p=8 k+5$; and $I(p)=2$, if $p=8 k+1$. 
Combining (4) and (5), we have the identity

$$
A^{2}(b)=R(p) \cdot p+2\left(\frac{b}{p}\right) \sqrt{p}\left(\sum_{a=1}^{p-1}\left(\frac{a}{p}\right) e\left(\frac{b a^{2}}{p}\right)\right)+\left(\frac{b}{p}\right) \sqrt{p} \sum_{a=1}^{p-1}\left(\frac{a+\bar{a}}{p}\right) .
$$

This proves formula (II) of Lemma 1.

Similarly, from (I) and (5) we can also prove that if $p=8 k+1$, then we have

$$
\begin{aligned}
A^{3}(b)= & 7\left(\frac{b}{p}\right) p^{\frac{3}{2}}+5 p\left(\sum_{a=1}^{p-1}\left(\frac{a}{p}\right) e\left(\frac{b a^{2}}{p}\right)\right)+3 p\left(\sum_{a=1}^{p-1}\left(\frac{a+\bar{a}}{p}\right)\right) \\
& +\left(\frac{b}{p}\right) \sqrt{p}\left(\sum_{a=1}^{p-1}\left(\frac{a}{p}\right) e\left(\frac{b a^{2}}{p}\right)\right)\left(\sum_{a=1}^{p-1}\left(\frac{a+\bar{a}}{p}\right)\right) .
\end{aligned}
$$

If $p=8 k+5$, then we have

$$
\begin{aligned}
A^{3}(b)= & -5\left(\frac{b}{p}\right) p^{\frac{3}{2}}+p\left(\sum_{a=1}^{p-1}\left(\frac{a}{p}\right) e\left(\frac{b a^{2}}{p}\right)\right)+3 p\left(\sum_{a=1}^{p-1}\left(\frac{a+\bar{a}}{p}\right)\right) \\
& +\left(\frac{b}{p}\right) \sqrt{p}\left(\sum_{a=1}^{p-1}\left(\frac{a}{p}\right) e\left(\frac{b a^{2}}{p}\right)\right)\left(\sum_{a=1}^{p-1}\left(\frac{a+\bar{a}}{p}\right)\right) .
\end{aligned}
$$

Lemma 2 Let $p$ be an odd prime with $p \equiv 1 \bmod 4, A(p)$ is defined as in the above lemma, and let

$$
N_{k}(p)=\sum_{b=1}^{p-1} A^{k}(b)
$$

Then we have the identities

$$
N_{1}(p)=0, \quad N_{2}(p)=R(p) p(p-1) \quad \text { and } \quad N_{3}(p)=3 p(p-1)\left(\sum_{a=1}^{p-1}\left(\frac{a+\bar{a}}{p}\right)\right)
$$

where $R(p)=-1$, if $p=8 k+5 ;$ and $R(p)=3$, if $p=8 k+1$.

Proof Note the trigonometric identity

$$
\sum_{m=0}^{p-1} e\left(\frac{n m}{p}\right)= \begin{cases}p, & \text { if }(p, n)=p \\ 0, & \text { if }(p, n)=1\end{cases}
$$

and

$$
\sum_{b=1}^{p-1}\left(\frac{b}{p}\right)=0
$$

From Lemma 1 and the properties of Gauss sums we have

$$
\sum_{b=1}^{p-1} A(b)=\sum_{b=1}^{p-1}\left(1+\sum_{a=1}^{p-1} e\left(\frac{b a^{4}}{p}\right)\right)=p-1+\sum_{a=1}^{p-1} \sum_{b=1}^{p-1} e\left(\frac{b a^{4}}{p}\right)=0,
$$




$$
\begin{aligned}
\sum_{b=1}^{p-1} A^{2}(b)= & \sum_{b=1}^{p-1} R(p) \cdot p+2 \sum_{b=1}^{p-1}\left(\frac{b}{p}\right) \sqrt{p}\left(\sum_{a=1}^{p-1}\left(\frac{a}{p}\right) e\left(\frac{b a^{2}}{p}\right)\right) \\
& +\sum_{b=1}^{p-1}\left(\frac{b}{p}\right) \sqrt{p} \sum_{a=1}^{p-1}\left(\frac{a+\bar{a}}{p}\right) \\
= & R(p) \cdot p(p-1)+2 \sqrt{p} \sum_{a=1}^{p-1}\left(\frac{a}{p}\right) \sum_{b=1}^{p-1}\left(\frac{b}{p}\right) e\left(\frac{b a^{2}}{p}\right) \\
= & R(p) \cdot p(p-1)+2 p \sum_{a=1}^{p-1}\left(\frac{a}{p}\right)^{3}=R(p) \cdot p(p-1) .
\end{aligned}
$$

Similarly, from (6) and (7) we have

$$
\sum_{b=1}^{p-1} A^{3}(b)=3 p(p-1)\left(\sum_{a=1}^{p-1}\left(\frac{a+\bar{a}}{p}\right)\right)
$$

where we have used the identity

$$
\sum_{b=1}^{p-1}\left(\sum_{a=1}^{p-1}\left(\frac{a}{p}\right) e\left(\frac{b a^{2}}{p}\right)\right)=\sum_{a=1}^{p-1}\left(\frac{a}{p}\right) \sum_{b=1}^{p-1} e\left(\frac{b a^{2}}{p}\right)=-\sum_{a=1}^{p-1}\left(\frac{a}{p}\right)=0 .
$$

Now Lemma 2 follows from (9), (10) and (11).

Lemma 3 Let $p$ be an odd prime with $4 \mid(p-1)$, then, for $p=8 k+5$, we have

$$
A^{4}(b)=-2 p A^{2}(b)+4 p A(b)\left(\sum_{a=1}^{p-1}\left(\frac{a+\bar{a}}{p}\right)\right)-9 p^{2}+p\left(\sum_{a=1}^{p-1}\left(\frac{a+\bar{a}}{p}\right)\right)^{2} .
$$

If $p=8 k+1$, then we have

$$
A^{4}(b)=6 p A^{2}(b)+4 p A(b)\left(\sum_{a=1}^{p-1}\left(\frac{a+\bar{a}}{p}\right)\right)-p^{2}+p\left(\sum_{a=1}^{p-1}\left(\frac{a+\bar{a}}{p}\right)\right)^{2}
$$

Proof First, for any integer $1 \leq b \leq p-1$, from (5) and (II) of Lemma 1 we have

$$
\begin{aligned}
A^{4}(b) & -2 R(p) p A^{2}(b)+R^{2}(p) p^{2} \\
= & p\left(2 \sum_{a=1}^{p-1}\left(\frac{a}{p}\right) e\left(\frac{b a^{2}}{p}\right)+\sum_{a=1}^{p-1}\left(\frac{a+\bar{a}}{p}\right)\right)^{2} \\
= & 4 p\left(\sum_{a=1}^{p-1}\left(\frac{a}{p}\right) e\left(\frac{b a^{2}}{p}\right)\right)^{2}+4 p\left(\sum_{a=1}^{p-1}\left(\frac{a}{p}\right) e\left(\frac{b a^{2}}{p}\right)\right)\left(\sum_{a=1}^{p-1}\left(\frac{a+\bar{a}}{p}\right)\right) \\
& +p\left(\sum_{a=1}^{p-1}\left(\frac{a+\bar{a}}{p}\right)\right)^{2} \\
= & 4 p\left(I(p) p+\left(\frac{b}{p}\right) \sqrt{p} \sum_{a=1}^{p-1}\left(\frac{a+\bar{a}}{p}\right)\right)+p\left(\sum_{a=1}^{p-1}\left(\frac{a+\bar{a}}{p}\right)\right)^{2}
\end{aligned}
$$




$$
\begin{aligned}
& +4 p\left(\sum_{a=1}^{p-1}\left(\frac{a}{p}\right) e\left(\frac{b a^{2}}{p}\right)\right)\left(\sum_{a=1}^{p-1}\left(\frac{a+\bar{a}}{p}\right)\right) \\
= & 4 I(p) p^{2}+4 p\left(\sum_{a=1}^{p-1}\left(\frac{a+\bar{a}}{p}\right)\right)\left(\left(\frac{b}{p}\right) \sqrt{p}+\sum_{a=1}^{p-1}\left(\frac{a}{p}\right) e\left(\frac{b a^{2}}{p}\right)\right) \\
& +p\left(\sum_{a=1}^{p-1}\left(\frac{a+\bar{a}}{p}\right)\right)^{2} \\
= & 4 I(p) p^{2}+4 p A(b)\left(\sum_{a=1}^{p-1}\left(\frac{a+\bar{a}}{p}\right)\right)+p\left(\sum_{a=1}^{p-1}\left(\frac{a+\bar{a}}{p}\right)\right)^{2} .
\end{aligned}
$$

From (12), the definitions of $R(p)$ and $I(p)$ we know that if $p=8 k+5$, then

$$
A^{4}(b)=-2 p A^{2}(b)+4 p A(b)\left(\sum_{a=1}^{p-1}\left(\frac{a+\bar{a}}{p}\right)\right)-9 p^{2}+p\left(\sum_{a=1}^{p-1}\left(\frac{a+\bar{a}}{p}\right)\right)^{2} .
$$

If $p=8 k+1$, then we have

$$
A^{4}(b)=6 p A^{2}(b)+4 p A(b)\left(\sum_{a=1}^{p-1}\left(\frac{a+\bar{a}}{p}\right)\right)-p^{2}+p\left(\sum_{a=1}^{p-1}\left(\frac{a+\bar{a}}{p}\right)\right)^{2}
$$

Now Lemma 3 follows from (13) and (14).

It is clear that our Lemma 3 obtained a fourth-order linear recurrence formula for the quartic Gauss sums $A(b)$.

\section{Proofs of the theorems}

Now we shall complete the proofs of our main results. First we prove Theorem 1 . If $p=$ $8 k+5$ is an odd prime, then, for any positive integer $n$, from (8) and the definition of $M_{n}(p)$ we have

$$
\begin{aligned}
& M_{n}(p)=\sum_{\substack{x_{1}=0 \\
x_{1}^{4}+x_{2}^{4}+\cdots+x_{n}^{4}=0 \bmod p}}^{p-1} \sum_{\substack{x_{2}=0 \\
x_{n}=0}}^{p-1} 1=\frac{1}{p} \sum_{b=0}^{p-1}\left(\sum_{x=0}^{p-1} e\left(\frac{b x^{4}}{p}\right)\right)^{n} \\
& =p^{n-1}+\frac{1}{p} \sum_{b=1}^{p-1}\left(\sum_{x=0}^{p-1} e\left(\frac{b x^{4}}{p}\right)\right)^{n}=p^{n-1}+\frac{1}{p} \sum_{b=1}^{p-1} A^{n}(b) \text {. }
\end{aligned}
$$

So from (15) and Lemma 2 we have

$$
\begin{aligned}
& U_{1}(p)=M_{1}(p)-1=\frac{1}{p} \sum_{b=1}^{p-1} A(b)=0, \\
& U_{2}(p)=M_{2}(p)-p=\frac{1}{p} \sum_{b=1}^{p-1} A^{2}(b)=-(p-1), \\
& U_{3}(p)=M_{3}(p)-p^{2}=\frac{1}{p} \sum_{b=1}^{p-1} A^{3}(b)=3(p-1)\left(\sum_{a=1}^{p-1}\left(\frac{a+\bar{a}}{p}\right)\right) .
\end{aligned}
$$


From Lemma 3 we have

$$
\begin{aligned}
U_{4}(p) & =M_{4}(p)-p^{3}=\frac{1}{p} \sum_{b=1}^{p-1} A^{4}(b) \\
& =-2 p U_{2}(p)+4 p U_{1}(p) B(p)-(p-1)\left(9 p-B(p)^{2}\right) \\
& =-7 p(p-1)+(p-1) B^{2}(p) .
\end{aligned}
$$

If $k \geq 5$, then from (15) and Lemma 3 we have

$$
\begin{aligned}
U_{k}(p)= & M_{k}(p)-p^{k-1}=\frac{1}{p} \sum_{b=1}^{p-1} A^{k}(b)=\frac{1}{p} \sum_{b=1}^{p-1} A^{k-4}(b) A^{4}(b) \\
= & \frac{1}{p} \sum_{b=1}^{p-1} A^{k-4}(b)\left(-2 p A^{2}(b)+4 p A(b)\left(\sum_{a=1}^{p-1}\left(\frac{a+\bar{a}}{p}\right)\right)\right) \\
& -\left(9 p-\left(\sum_{a=1}^{p-1}\left(\frac{a+\bar{a}}{p}\right)\right)^{2}\right) \sum_{b=1}^{p-1} A^{k-4}(b) \\
= & -2 p U_{k-2}(p)+4 p\left(\sum_{a=1}^{p-1}\left(\frac{a+\bar{a}}{p}\right)\right) U_{k-3}(p) \\
& -\left(9 p^{2}-p\left(\sum_{a=1}^{p-1}\left(\frac{a+\bar{a}}{p}\right)\right)^{2}\right) U_{k-4}(p) .
\end{aligned}
$$

Now Theorem 1 follows from formulas (16)-(20).

If $p=8 k+1$, then from (15) and Lemma 2 we have

$$
\begin{aligned}
& U_{1}(p)=M_{1}(p)-1=\frac{1}{p} \sum_{b=1}^{p-1} A(b)=0, \\
& U_{2}(p)=M_{2}(p)-p=\frac{1}{p} \sum_{b=1}^{p-1} A^{2}(b)=3(p-1), \\
& U_{3}(p)=M_{3}(p)-p^{2}=\frac{1}{p} \sum_{b=1}^{p-1} A^{3}(b)=3(p-1)\left(\sum_{a=1}^{p-1}\left(\frac{a+\bar{a}}{p}\right)\right) .
\end{aligned}
$$

From Lemma 3 we also have

$$
\begin{aligned}
U_{4}(p) & =M_{4}(p)-p^{3}=\frac{1}{p} \sum_{b=1}^{p-1} A^{4}(b) \\
& =6 p U_{2}(p)+4 p U_{1}(p)\left(\sum_{a=1}^{p-1}\left(\frac{a+\bar{a}}{p}\right)\right)-(p-1)\left(p-\left(\sum_{a=1}^{p-1}\left(\frac{a+\bar{a}}{p}\right)\right)^{2}\right) \\
& =17 p(p-1)+(p-1) B^{2}(p) .
\end{aligned}
$$


If $k \geq 5$, then from (15) and Lemma 3 we also have

$$
\begin{aligned}
U_{k}(p)= & M_{k}(p)-p^{k-1}=\frac{1}{p} \sum_{b=1}^{p-1} A^{k}(b)=\frac{1}{p} \sum_{b=1}^{p-1} A^{k-4}(b) A^{4}(b)=6 p U_{k-2}(p) \\
& +4 p\left(\sum_{a=1}^{p-1}\left(\frac{a+\bar{a}}{p}\right)\right) U_{k-3}(p)-\left(p^{2}-p\left(\sum_{a=1}^{p-1}\left(\frac{a+\bar{a}}{p}\right)\right)^{2}\right) U_{k-4}(p) .
\end{aligned}
$$

Now Theorem 2 follows from formulas (21)-(25).

\section{Competing interests}

The authors declare that there is no conflict of interests regarding the publication of this paper.

\section{Authors' contributions}

WPZ put forward the main ideas, and SMS carried on the actual operation and finishing process. All authors read and approved the final manuscript.

\section{Acknowledgements}

The authors would like to thank the referees for their very helpful and detailed comments, which have significantly improved the presentation of this paper. This work is supported by the N.S.F. (11371291) of P.R. China.

Received: 16 November 2016 Accepted: 1 February 2017 Published online: 07 February 2017

\section{References}

1. Weil, A: On some exponential sums. Proc. Natl. Acad. Sci. USA 34, 204-207 (1948)

2. Zhang, W, Liu, H: On the general Gauss sums and their fourth power mean. Osaka J. Math. 42, 189-199 (2005)

3. Yang, QH, Tang, M: On the addition of squares of units and nonunits modulo $n$. J. Number Theory 155, 1-12 (2015)

4. Chowla, S, Cowles, J, Cowles, M: On the number of zeros of diagonal cubic forms. J. Number Theory 9, 502-506 (1977)

5. Sander, JW: On the addition of units and nonunits mod $m$. J. Number Theory 129, 2260-2266 (2009)

6. Golomb, S: On the algebraic construction for Costas arrays. J. Comb. Theory, Ser. A 37, 13-21 (1984)

7. Cohen, SD, Zhang, W: Sums of two exact powers. Finite Fields Appl. 8, 471-477 (2002)

8. Zhang, W, Li, H: Elementary Number Theory. Shaanxi Normal University Press, Xi'an (2013)

9. Apostol, TM: Introduction to Analytic Number Theory. Springer, New York (1976)

\section{Submit your manuscript to a SpringerOpen ${ }^{\circ}$ journal and benefit from:}

- Convenient online submission

Rigorous peer review

- Immediate publication on acceptance

- Open access: articles freely available online

- High visibility within the field

- Retaining the copyright to your article 